TRANSACTIONS OF THE

AMERICAN MATHEMATICAL SOCIETY

Volume 224, Number 1, 1976

\title{
MAXIMAL CHAINS OF PRIME IDEALS IN INTEGRAL EXTENSION DOMAINS. I
}

\author{
BY
}

\author{
L. J. RATLIFF, JR. AND S. MCADAM(1)
}

ABSTRACT. Let $(R, M)$ be a local domain, let $k$ be a positive integer, and let $Q$ be a prime ideal in $R_{k}=R\left[X_{1}, \ldots, X_{k}\right]$ such that $M R_{k} \subset Q$. Then the following statements are equivalent: (1) There exists an integral extension domain of $R$ which has a maximal chain of prime ideals of length $n$. (2) There exists a minimal prime ideal $z$ in the completion of $R$ such that depth $z=n$. (3) There exists a minimal prime ideal $w$ in the completion of $\left(R_{k}\right)_{Q}$ such that depth $w=n+k$ - depth $Q$. (4) There exists an integral extension domain of $\left(R_{k}\right)_{Q}$ which has a maximal chain of prime ideals of length $n+k-$ depth $Q$. (5) There exists a maximal chain of prime ideals of length $n+k-$ depth $Q$ in $\left(R_{k}\right)_{Q}$. (6) There exists a maximal chain of prime ideals of length $n+1$ in $R\left[X_{1}\right]_{\left(M, X_{1}\right)}$.

1. Introduction. In [6, Theorem 3.6], it is shown that if $(R, M)$ is a local domain, then $R_{1}=R\left[X_{1}\right]$ is catenary if and only if $R_{k}=R\left[X_{1}, \ldots, X_{k}\right]$ is catenary, for all $k \geq 0$. This result is closely related to the equivalence of the following statements (see (1.1) for the definitions): $R$ satisfies the second chain condition for prime ideals (s.c.c.); $R$ satisfies the altitude formula; and, $R$ is quasi-unmixed [6, Theorem 3.6]. Thus it is of some interest.

The original goal of the authors in starting this paper was to see how the lengths of maximal chains of prime ideals in $R_{1}$ compare to the lengths of maximal chains of prime ideals in $R_{k}$ (without assuming that $R_{1}$ is catenary). The answer we obtained is included in the result mentioned at the start of this paper, and, as is seen by that result, it is closely related to the lengths of maximal chains of prime ideals in integral extension domains of $R$ and to the depths of the minimal prime ideals in the completion of $R$. (This result is given in (2.3), (2.5), (2.9), and, more specifically (2.14).)

Received by the editors May 28, 1975.

AMS (MOS) subject classifications (1970). Primary 13A15, 13B20, 13C15; Secondary $13 \mathrm{~B} 25$.

Key words and phrases. Altitude formula, catenary ring, catenary chain conjecture, chain condition for prime ideals, chain conjecture, completion of a local ring, depth conjecture, first chain condition for prime ideals, integral extension, local ring, maximal chain of prime ideals, Noetherian ring, polynomial extension ring, quasi-unmixed local ring, second chain condition for prime ideals, semilocal ring, unmixed local ring, upper conjecture.

(1) Research supported in part by NSF-MCS 76-06009. 
After the main theorem (the above six equivalent statements) is proved, the rest of the paper is devoted to showing that a large number of important known results follow as easy corollaries. Among these are: (a) $R$ satisfies the s.c.c. if and only if some $\left(R_{k}\right)_{Q}$ is catenary if and only if all $\left(R_{k}\right)_{Q}$ satisfy the s.c.c. (2.16) and (2.17); (b) $R$ satisfies the s.c.c. if and only if $R$ is quasi-unmixed (2.19); (c) if $R$ satisfies the s.c.c., then, for each prime ideal $p$ in $R$, every local domain which is a locality over $R / p$ satisfies the s.c.c. (2.20) (in particular, this holds for $R / p$ and $R_{p}$ (2.26)); (d) the above mentioned [6, Theorem 3.6] (2.18); (e) two equivalences to the upper conjecture (2.22) (see (2.21)); and, (f) the relationship between lengths of maximal chains of prime ideals in $\left(R_{k}\right)_{Q}$ and in $R\left[X_{1}\right]_{\left(M, X_{1}\right)}$ (2.9.2) (our original goal). (A number of quite deep theorems are used to prove the equivalence of the above six statements, so the authors do not claim that this method affords easier proofs than those originally given for the above-mentioned corollaries. However, once it is proved that the above six statements are equivalent, the corollaries are nearly obvious.)

All rings in this paper are assumed to be commutative rings with an identity element. The undefined terminology is the same as that in [5]. We mention, in particular, that $A \subset B$ means that the set $A$ is a proper subset of the set $B$.

We close this introduction with the following less well-known definitions, since they are frequently used in the remainder of this paper.

(1.1) Definitions. Let $A$ be an integral domain, and let $a=$ altitude $A$ $<\infty$.

(1.1.1) $A$ satisfies the first chain condition for prime ideals (f.c.c.) in case every maximal chain of prime ideals in $A$ has length $=a$.

(1.1.2) $A$ is catenary in case, for each pair of prime ideals $P \subset Q$ in $A$, $(A / P)_{Q / P}$ satisfies the f.c.c.

(1.1.3) $A$ satisfies the second chain condition for prime ideals (s.c.c.) in case every integral extension domain of $A$ satisfies the f.c.c.

(1.1.4) $A$ satisfies the chain condition for prime ideals (c.c.) in case, for each pair of prime ideals $P \subset Q$ in $A,(A / P)_{Q / P}$ satisfies the s.c.c.

(1.1.5) $A$ is quasi-unmixed (resp., unmixed) in case $A$ is semilocal and every minimal (resp., every) prime divisor of zero in the completion of $A$ has depth $=a$.

(1.1.6) $A$ satisfies the altitude formula in case, for each finitely-generated integral domain $B$ over $A$ and for each prime ideal $P$ in $B$, altitude $B_{P}+$ $\operatorname{trd}(B / P) /(A /(P \cap A))=$ altitude $A_{P \cap A}+\operatorname{trd} B / A$, where $\operatorname{trd} C / D$ denotes the transcendence degree of the quotient field of the integral domain $C$ over the quotient field of its subdomain $D$.

A number of facts concerning these definitions are given in [8, Remarks 
2.22-2.25 and 3.7-3.8]. We mention at this point only that, for a local domain $R, R$ is catenary if and only if $R$ satisfies the f.c.c.

2. Main results. We will be greatly concerned with the lengths of maximal chains of prime ideals in a ring. Therefore, since we use the expression so often, we adopt the following abbreviation.

(2.1) Definition. It will be said that a ring $A$ has a mcpil $n$ in case there exists a maximal chain of prime $i$ deals of length $n$ in $A$ (that is, a chain of prime ideals $p_{0} \subset p_{1} \subset \cdots \subset p_{n}$ such that $p_{0}$ is minimal, $p_{n}$ is maximal, and height $\left.p_{i} / p_{i-1}=1(i=1, \ldots, n)\right)$.

The following lemma combines, for future reference, two known and quite useful results [1, Corollary 4] and [2, Theorem 1.10]. Only the last sentence of the lemma is new.

(2.2) Lemma (CF. [1, Corollary 4] AND [2, Theorem 1.10]). Let $A \subseteq$ $B$ be integral domains such that $A$ is Noetherian and $B$ is integral over $A$. Assume that $B$ has a mcpil $n$. Then there exists a principal integral extension domain $C=A[c]$ of $A$ which has a mcpil $n$, say $(0) \subset p_{1} \subset \cdots \subset p_{n}$, such that height $p_{i}=i=$ height $p_{i} \cap A(i<n)$. Further, $c$ may be chosen so as to satisfy either of the conditions: $c \in p_{n}$; or, $c \notin p_{n}$.

Proof. Let $N$ be a maximal ideal in $B$ such that there exists a mcpil $n$ in $B_{N}$, and let $M=N \cap A$. Then it follows from [2, Theorem 1.10] (applied to $A_{M}$ ) that there exists a finite integral extension domain $D$ of $A$ which has a mcpil $n$, say (0) $\subset Q_{1} \subset \cdots \subset Q_{n}$. Therefore, there exists a maximal chain of prime ideals (0) $\subset q_{1} \subset \cdots \subset q_{n-1} \subset q_{n}=Q_{n}$ in $D$ such that height $q_{i}=i=$ height $q_{i} \cap A(i<n)$ [1, Corollary 4]. Let $c \in q_{n}$ such that $c$ is not in any other maximal ideal in $D$ which lies over $q_{n} \cap A$. Then it is readily seen that $C=A[c]$ has a mcpil $n$ (namely, (0) $\subset p_{1} \subset \cdots \subset p_{n-1} \subset p_{n}$, where $p_{i}=$ $\left.q_{i} \cap C\right)$ such that height $p_{i}=i=$ height $p_{i} \cap A(i<n)$ and $c \in p_{n}$. Then also, $C=A[c-1]$ has a mcpil $n$ and $c-1 \notin p_{n}$. Q.E.D.

The following result is the first part of the main theorem in this paper.

(2.3) THEOREM. Let $R$ be a semilocal domain, and let $n$ be a positive integer. Then there exists an integral extension domain $D$ of $R$ which has a mcpil $n$ if and only if there exists a minimal prime ideal $z$ in the completion $R^{*}$ of $R$ such that depth $z=n$.

Proof. If $R$ is local, then it is known [6, Proposition 3.5] that there exists a height one maximal ideal in the integral closure $R^{\prime}$ of $R$ if and only if . there exists a depth one minimal prime ideal in $R^{*}$. Therefore, for the semilocal case, there exists a depth one minimal prime ideal in $R^{*}$ if and only if there exists a depth one minimal prime ideal in $R_{M}^{*}$, for some maximal ideal $M^{*}$ in 
$R^{*}$, if and only if, by [6, Proposition 3.5], there exists a height one maximal ideal in the integral closure of $R_{M^{*} \cap R}$ (since the completion of $R_{M^{*} \cap R}$ is $R_{M^{*}}^{*}$ ) if and only if there exists a height one maximal ideal in $R^{\prime}$. Therefore, assume that $n>1$, and assume that the lemma holds for $n-1$.

Assume first that such a $D$ exists. Then, by (2.2), it may be assumed that $D=R[c]$ and (0) $\subset Q_{1} \subset \cdots \subset Q_{n}$ is a mcpil $n$ in $D$ such that height $Q_{i}=i$ $=$ height $Q_{i} \cap R(i<n)$. Let $q=Q_{1} \cap R$, so $D / Q_{1}=(R / q)\left[c^{\prime}\right]$ (with $c^{\prime}=$ $c+Q_{1}$ ) has a mcpil $n-1$. Therefore, by the case $n-1$, there exists a depth $n-1$ minimal prime ideal in the completion $(R / q)^{*} \cong R^{*} / q R^{*}$ of $R / q$. Therefore $q R^{*}$ has a minimal prime divisor of depth $n-1$, say $q^{*}$, and height $q^{*}=$ height $q=1$. Then there exists a minimal prime ideal $z$ in $R^{*}$ such that $z \subset q^{*}$, so depth $z=n$ (since $R^{*} / z$ is a complete local domain, so is catenary (1.1.2), hence $n=1+\operatorname{depth} q^{*}=$ height $q^{*} / z+\operatorname{depth} q^{*} / z=$ altitude $R^{*} / z=\operatorname{depth} z$ ).

Conversely, assume that $z$ is a depth $n$ minimal prime ideal in $R^{*}(n>1)$. Then there exists a height one prime ideal $p^{*}$ in $R^{*}$ such that $z \subset p^{*}$ and $p=$ $p^{*} \cap R \neq(0)$. (For, there exist only a finite number of prime ideals $p^{*}$ in $R^{*}$ such that $z \subset p^{*}$ and height $p^{*} / z=1<$ height $p^{*}\left[1\right.$, Theorem 1], so let $p_{1}^{*}$, $\ldots, p_{k}^{*}$ be these. (No $p_{i}^{*}$ is maximal, since $n>1$ and $R^{*} / z$ is local.) Then, with $J$ the Jacobson radical of $R, J \nsubseteq \cup\left(p_{i}^{*} \cap R\right)$, so let $b \in J, \notin \cup\left(p_{i}^{*} \cap R\right)$. Then $(z, b) R^{*}$ has a height one prime divisor $p^{*}$ (by the choice of $b$ and the principal ideal theorem in $\left.R^{*} / z\right)$, so $p^{*} \supset z$ and $p^{*} \cap R \neq(0)$.) Then $p^{*}$ is a minimal prime divisor of $p R^{*}$; hence height $p=1[5,(22.9)]$. Now, as above, depth $p^{*}$ $=$ depth $z-1=n-1$. Now, if depth $p=n-1$, then $R$ has a mcpil $n$. Therefore assume that depth $p>n-1$ (depth $\left.p \geqslant \operatorname{depth} p^{*}\right)$. Then in $(R / p)^{*} \cong \cdot$ $R^{*} / p R^{*}$ there exists a depth $n-1$ minimal prime ideal (namely, $p^{*} / p R^{*}$ ), so, by the case $n-1$ and (2.2), there exists an integral extension domain $D^{\prime}=(R / p)$ [ $\left.c^{\prime}\right]$ of $R / p$ which has a mcpil $n-1$. Thus there exists an integral extension domain $D=R[c]$ of $R$ which has a prime ideal $q$ such that $q \cap R=p$ and such that $D / q=D^{\prime}$. Then height $q=1$, since height $p=1$, so $D$ has a mcpil $n$ (since $D^{\prime}$ has a mcpil $\left.n-1\right)$. Q.E.D.

(2.4) REMARK. The case $n=1$ of (2.3) was essentially proved in [6, Proposition 3.5], as was mentioned at the start of the proof of (2.3). A hoped for generalization of the case $n=1$ is:

There exists a depth $n$ minimal prime ideal in the completion $R^{*}$ of $R$ if and only if there exists a height $n$ maximal ideal in the integral closure $R^{\prime}$ of $R$.

This generalization is, in fact, equivalent to the chain conjecture (that is, the integral closure of a semilocal domain satisfies the c.c. (1.1.4)).

Proof. Assume first that the chain conjecture holds. If there exists a depth $n$ minimal prime ideal in $R^{*}$, then, by (2.3), there exists an integral extension 
domain $D$ of $R$ which has a mcpil $n$. Then $D\left[R^{\prime}\right]$ is an integral extension domain of $R^{\prime}$ which has a mcpil $n$ (by the going-up theorem), so, since $R^{\prime}$ satisfies the c.c., there exists a height $n$ maximal ideal in $R^{\prime}$. (And, if there exists a height $n$ maximal ideal in $R^{\prime}$, then there exists a depth $n$ minimal prime ideal in $R^{*}$, by (2.3).) Hence (*) holds.

For the converse, to prove that the chain conjecture holds, it suffices to prove that if $H$ is a Henselian local domain, then $H$ satisfies the s.c.c. (1.1.3) [9, (2.4)]. For this, if (*) holds, then, for each minimal prime ideal $z$ in the completion of $H$, depth $z=$ altitude $H$ (since there is only one maximal ideal in the integral closure of $H$ ). Therefore, $H$ is quasi-unmixed (1.1.5); hence $H$ satisfies the s.c.c. [6, Theorem 3.1]. Q.E.D.

To prove the second part of our main theorem we need two facts. The first of these is that a transcendental extension domain of a Noetherian domain $A$ satisfies the altitude formula relative to $A$ [11, Proposition 2, p. 326].

Also, to prove (2.5), we need the following result: If $C$ is a class of local domains $R$ such that each $R \in C$ is a locality over some complete local domain, then each $R \in C$ is analytically unramified and unmixed (1.1.5). This follows from [3, Proposition 4]. (It is straightforward to check that the hypotheses of [3, Proposition 4] are satisfied by $C$.)

(2.5) THEOREM. Let $(R, M)$ be a local ring, let $n$ and $k$ be positive integers, and let $Q$ be a prime ideal in $R_{k}=R\left[X_{1}, \ldots, X_{k}\right]$ such that $Q \cap R=M$. Then there exists a depth $n$ minimal prime ideal in the completion $\left(R^{*}, M^{*}\right)$ of $R$ if and only if there exists a minimal prime ideal $w$ in the completion $L^{*}$ of $L=\left(R_{k}\right)_{Q}$ such that depth $w=n+k-$ depth $Q$.

Proof. Let $I=R^{*}\left[X_{1}, \ldots, X_{k}\right]$. Then, by [7, Lemma 3.2], $Q^{*}=$ $Q I$ is a prime ideal and $L$ is a dense subspace of $S=I_{Q^{*}}$, so $S^{*}=L^{*}$, where $S^{*}$ is the completion of $S$. Further, depth $Q^{*}=\operatorname{depth} Q$, since $I / Q^{*}=R_{k} / Q$.

Assume first that $z$ is a depth $n$ minimal prime ideal in $R^{*}$. Then $z S$ is a minimal prime ideal in $S$ and depth $z S=n+k-\operatorname{depth} Q^{*}$, as will now be shown. Namely, $z I \subseteq M^{*} I \subseteq Q^{*}$, so $Q^{*} / z I$ is a prime ideal in $I / z I \cong\left(R^{*} / z\right)$ $\left[X_{1}, \ldots, X_{k}\right]$ which lies over $M^{*} / z$, so height $Q^{*} / z I+\operatorname{trd}\left(I / Q^{*}\right) /\left(R^{*} / M^{*}\right)=$ height $M^{*} / z+k$, by the altitude formula (1.1.6) [11, Proposition 2, p. 326]; that is, depth $z S=$ depth $z+k-\operatorname{depth} Q^{*}$, since depth $Q^{*}=$ altitude $I / Q^{*}=$ $\operatorname{trd}\left(I / Q^{*}\right) /\left(R^{*} / M^{*}\right)[5,(14.6)]$ (since $R^{*} / M^{*}$ is a field). Therefore, there exists a minimal prime ideal $w$ in $L^{*}=S^{*}$ such that depth $w=n+k-\operatorname{depth} Q$, since depth $Q=$ depth $Q^{*}$, by the preceding paragraph. (Any minimal prime divisor $w$ of $(z S) L^{*}$ will do, by [3, Proposition 4].)

Conversely, let $w$ be a minimal prime ideal in $L^{*}=S^{*}$, and let depth $w=$ $d$. Let $q=w \cap S$, so $q$ is a minimal prime ideal in $S$ and $w$ is a minimal prime 
divisor of $q S^{*}$. Hence, since $S / q$ is unmixed, by the comment preceding this theorem, depth $q=$ depth $w=d$. Also, $z=q \cap R^{*}$ is a minimal prime ideal and $q=z S$. Therefore, by the preceding paragraph, $d=\operatorname{depth} q=\operatorname{depth} z S=$ depth $z+k$ - depth $Q$. Q.E.D.

(2.6) CoRollary. Let $(R, M), k, Q$, and $L$ be as in (2.5), and assume that $R$ is an integral domain. Then there exists an integral extension domain of $R$ which has a mcpil $n$ if and only if there exists an integral extension domain of $L$ which has a mcpil $n+k$ - depth $Q$.

Proof. This follows immediately from (2.3) and (2.5). Q.E.D.

It follows from (2.6) that there exists an integral extension domain of $R$ which has a mcpil $n$ if and only if there exists an integral extension domain of $R\left[X_{1}, \ldots, X_{k}\right]_{\left(M, X_{1}, \ldots, X_{k}\right)}$ which has a mcpil $n+k$. This fact will be considerably sharpened in (2.14) below. For the present, it suffices to emphasize that (2.6) depends only on depth $Q$, and not on the ideal $Q$ itself. It should also be noted, in particular, that, by (2.6), if there exists a mcpil $m$ in $\left(R_{k}\right)_{Q}$ $\left(M R_{k} \subset Q\right)$, then there exists an integral extension domain of $R$ which has a mcpil $m-k+$ depth $Q$.

We need one further result to complete the proof of our main theorem. The needed result is a weak version of a result given in [10,(3.1)]. However, since a proof of the weak version of the result is not long, we include it at this point.

(2.7) Proposition. Let $(R, M)$ and $(S, N)$ be local domains such that $S$ is a finite integral extension of $R$. Then there exists a mcpil $n$ in $R$ if and only if there exists a mcpil $n$ in $S$.

Proof. Assume that there exists a mcpil $n$ in $S$. Then, by [1, Corollary 4], there exists a mcpil $n$ in $S$, say (0) $\subset p_{1} \subset \cdots \subset p_{n}=N$ such that height $p_{i}=i=$ height $p_{i} \cap R(i<n)$. Then (0) $\subset p_{1} \cap R \subset \cdots \subset p_{n-1} \cap R \subset N \cap$ $R=M$ is a mcpil $n$ in $R$, since depth $p_{n-1} \cap R=\operatorname{depth} p_{n-1}=1$.

The converse follows from the going-up theorem [5, (10.9)]. Q.E.D.

It is now expedient to introduce notation for the set of lengths of maximal chains of prime ideals in localizations of transcendental extensions of a ring. We do so in the following definition.

(2.8) Definition. Let $A$ be a ring, $k$ a nonnegative integer and $Q$ a prime ideal in $A_{k}=A\left[X_{1}, \ldots, X_{k}\right]\left(A_{0}=A\right)$. Then $W(k, Q)=\{n$; there exists a mcpil $n$ in $\left(A_{k}\right)_{Q}$.

The following theorem completes the proof of the main result in this paper. (The main theorem is summarized in (2.14) below.) A number of important known results follow easily from it, as will be seen in the subsequent corollaries. 
(2.9.1) was proved in [1, Theorem 6]. We include it here for completeness.

(2.9) THEOREM. Let $(R, M)$ be a local domain, let $k$ be a positive integer, and let $Q$ be a prime ideal in $R_{k}$ such that $Q \cap R=M$. Then the following statements hold:

(2.9.1) If $Q=M R_{k}$, then $W(k, Q)=W(0, M)$.

(2.9.2) If $Q \supset M R_{k}$, then $W(k, Q)=W^{\prime}(k, Q)=W^{\prime \prime}(k, Q)$, where $W^{\prime}(k, Q)$ $=\left\{n\right.$; there exists a mcpil $n$ in some integral extension domain of $\left.\left(R_{k}\right)_{Q}\right\}$ and $W^{\prime \prime}(k, Q)=\left\{m+k-\operatorname{depth} Q-1 ; m \in W\left(1,\left(M, X_{1}\right)\right)\right\}$.

Proof. (2.9.1) was proved in [1, Theorem 6].

(2.9.2) For $k=1$ and $Q=\left(M, X_{1}\right) R\left[X_{1}\right], W(k, Q)=W^{\prime \prime}(k, Q)=$ $W\left(1,\left(M, X_{1}\right)\right)$, so it suffices to prove that $W(k, Q)=W^{\prime}(k, Q)$ in this case. Assume temporarily that this is known. Then $W^{\prime}(k, Q)=W^{\prime \prime}(k, Q)$ in general, since $n \in W^{\prime}(k, Q)$ if and only if there exists an integral extension domain of $\left(R_{k}\right)_{Q}$ which has a mcpil $n$ if and only if (by (2.6)) there exists an integral extension domain of $R$ which has a mcpil $n-k+$ depth $Q$ if and only if (by (2.6) and assumption) $n-k+$ depth $Q+1 \in W\left(1,\left(M, X_{1}\right)\right)$ if and only if $n \in W^{\prime \prime}(k, Q)$. Therefore it suffices to prove that $W\left(1,\left(M, X_{1}\right)\right)=W^{\prime}\left(1,\left(M, X_{1}\right)\right)$ and $W(k, Q)$ $=W^{\prime}(k, Q)\left(k>0\right.$ and $\left.M R_{k} \subset Q\right)$. However, the proofs of both of these are essentially the same, so it will be shown that $W(k, Q)=W^{\prime}(k, Q)$.

For this, it is clear that $W(k, Q) \subseteq W^{\prime}(k, Q)$. For the opposite inclusion, let $d=\operatorname{depth} Q$. Then $d=\operatorname{trd}\left(R_{k} / Q\right) /(R / M)[5,(14.6)]$, so renumber the $X_{i}$ such that $X_{1}, \ldots, X_{d}$ modulo $Q$ are a transcendence basis for $R_{k} / Q$ over $R / M$. Then $Q \cap R_{d}=M R_{d}\left[7\right.$, Lemma 4.2] (where $R_{d}=R\left[X_{1}, \ldots, X_{d}\right]$ ). Also, with $A=\left(R_{d}\right)_{M R_{d}}$ and $B=A\left[X_{d+1}, \ldots, X_{k}\right],\left(R_{k}\right)_{Q}=B_{P}$, where $P=$ $Q\left(R_{k}\right)_{Q} \cap B$, and $P$ is a maximal ideal in $B$ (since $P \cap A=M A$ and $B / P$ is algebraic over the field $A / M A)$. Let $D=A\left[X_{d+1}, \ldots, X_{k-1}\right]$ ( $=A$, if $d=k-1$; $d \leqslant k-1$, since $Q \supset M R_{k}$ ), and let $N=P \cap D$, so $N$ is a maximal ideal in $D$.

Now assume that $n \in W^{\prime}(k, Q)$; that is, assume that there exists an integral extension domain of $\left(R_{k}\right)_{Q}$ which has a mcpil $n$. Then, since $B \cong D[X]$ and $P$ and $N=P \cap D$ are maximal ideals, and since $\left(R_{k}\right)_{Q}=B_{P}$, there exists an integral extension domain of $S=D_{N}$ which has a mcpil $n-1(2.6)$. Therefore, by (2.2), there exists a principal integral extension domain $S[c]$ of $S$ which has a mopil $n-1$ such that $c \in p_{n-1}$ (where (0) $\subset p_{1} \subset \cdots \subset p_{n-1}$ is the chain). Hence, considering the preimage of this chain in $S[X]$, there exists a mcpil $n$ in $S[X]_{(N S, X)} \cong D[X]_{(N, X)}$.

Since $B \cong D[X]$, let $P^{\prime}$ be the maximal ideal in $D[X]$ which corresponds to $P$, so $P^{\prime} \cap D=P \cap D=N$. Also, $P^{\prime}=(N, f) D[X]$, for some monic polynomial $f \in D[X]$, so, since $T=D[f]_{(N, f)} \cong D[X]_{(\dot{N}, X)}$, there exists a mcpil $n$ 
in $T$. Then $D[X]_{P^{\prime}}$ is integral over $T$ (since $P^{\prime}=P^{\prime \prime} D[X]$, where $P^{\prime \prime}=$ $\left.P^{\prime} \cap D[f]=(N, f) D[f]\right)$, so there exists a mcpil $n$ in $D[X]_{P^{\prime}}(2.7)$; hence there exists a mcpil $n$ in $\left(R_{k}\right)_{Q}=B_{P} \cong D[X]_{P^{\prime}}$. Therefore $n \in W(k, Q)$. Q.E.D.

(2.10) Corollary. Let $(R, M)$ be a local domain. Then $m \in$ $W\left(1,\left(M, X_{1}\right)\right)$ if and only if $m+k-1 \in W\left(k,\left(M, X_{1}, \ldots, X_{k}\right)\right)$.

PRoof. Let $Q=\left(M, X_{1}, \ldots, X_{k}\right) R_{k}$. Then, by (2.9.2),

$$
\begin{aligned}
W(k, Q)=W^{\prime \prime} & =\left\{m+k-\operatorname{depth} Q-1 ; m \in W\left(1,\left(M, X_{1}\right)\right)\right\} \\
& =\left\{m+k-1 ; m \in W\left(1,\left(M, X_{1}\right)\right)\right\},
\end{aligned}
$$

since depth $Q=0$. Q.E.D.

(2.11) Corollary. Let $(R, M)$ and $k$ be as in (2.9), and let $P$ and $Q$ be prime ideals in $R_{k}$ such that depth $P=\operatorname{depth} Q$ and $P \cap R=M=Q \cap R$. Then $W(k, P)=W(k, Q)$.

Proof. This follows immediately from (2.9). Q.E.D.

The following corollary contains (2.10) and (2.11) as special cases.

(2.12) CoROllary. Let $(R, M)$ be a local domain, let $k \leqslant n$ be positive integers, let $P$ be a prime ideal in $R_{k}$ such that $M R_{k} \subset P$, and let $Q$ be a prime ideal in $R_{n}$ such that $M R_{n} \subset Q$. Then $W(n, Q)=\{i+n-\operatorname{depth} Q-k+$ depth $P ; i \in W(k, P)\}$. In particular, if depth $P=\operatorname{depth} Q$, then $W(n, Q)=$ $\{i+n-k ; i \in W(k, P)\}$.

Proof. By (2.9.2), $j \in W(n, Q)$ if and only if $j=m+n-\operatorname{depth} Q-1$ and $i \in W(k, P)$ if and only if $i=m+k-\operatorname{depth} P-1$, where $m \in$ $W\left(1,\left(M, X_{1}\right)\right)$. The conclusion follows from this. Q.E.D.

The following corollary will be sharpened in (2.16)-(2.18) below.

(2.13) COROLlaRY. The following statements are equivalent for a local domain $(R, M)$ :

(2.13.1) $R\left[X_{1}\right]_{\left(M, X_{1}\right)}$ is catenary.

(2.13.2) There exists a positive integer $k$ and a prime ideal $Q$ in $R_{k}$ such that $M R_{k} \subset Q$ and $\left(R_{k}\right)_{Q}$ is catenary.

(2.13.3) For all integers $k \geqslant 0$, and for all prime ideals $Q$ in $R_{k}$ such that $Q \cap R=M,\left(R_{k}\right)_{Q}$ is catenary.

Proof. It is clear that (2.13.3) $\Rightarrow(2.13 .1) \Rightarrow(2.13 .2)$. Finally, if (2.13.2) holds, then $W(k, Q)$ is a one-element set; hence $W\left(1,\left(M, X_{1}\right)\right)$ is a one-element set (2.9.2), and so, for all $n>0$ and for all prime ideals $P$ in $R_{n}$ such that $M R_{n}$ $C P, W(n, P)$ is a one-element set (2.9.2); hence $\left(R_{n}\right)_{P}$ is catenary. Further, $R$ is catenary if and only if $\left(R_{n}\right)_{M R_{n}}$ is catenary (2.9.1). Hence, since the catenary 
property is inherited by quotient rings and $\left(R_{n}\right)_{M R_{n}}$ is a quotient ring of $\left(R_{n}\right)_{P}$, (2.13.2) implies (2.13.3). Q.E.D.

Before giving further corollaries of (2.9), it will be helpful at this point to summarize the main theorem.

(2.14) Summary of MAIN THEOREM. Let $(R, M)$ be a local domain, let $k$ be a positive integer, let $Q$ be a prime ideal in $R_{k}$ such that $M R_{k} \subset Q$, and let $S=\left(R_{k}\right)_{Q}$. Then the following statements are equivalent:

(2.14.1) There exists an integral extension domain of $R$ which has a mcpil $n$.

(2.14.2) There exists a minimal prime ideal $z$ in the completion of $R$ such that depth $z=n$.

(2.14.3) There exists a minimal prime ideal $w$ in the completion of $S$ such that depth $w=n+k-$ depth $Q$.

(2.14.4) There exists an integral extension domain of $S$ which has a mcpil $n+k-$ depth $Q$.

(2.14.5) There exists a mcpil $n+k$-depth $Q$ in $S$.

(2.14.6) There exists a mcpil $n+1$ in $R\left[X_{1}\right]_{\left(M, X_{1}\right)}$.

Proof. (2.14.1) $\Longleftrightarrow(2.14 .2)$, by (2.3). (2.14.2) $\Longleftrightarrow$ (2.14.3), by (2.5). (2.14.3) $\Longleftrightarrow(2.14 .4)$, by (2.3). (2.14.4) $\Longleftrightarrow(2.14 .5)$, by (2.9.2). (2.14.5) $\Longleftrightarrow$ (2.14.6), by (2.12). Q.E.D.

(2.14.1) $\Longleftrightarrow(2.14 .6)$ was given in [2, Theorem 1.8]

The following summary is included for the sake of completeness.

(2.15) Summary. With $(R, M)$ and $k$ as in (2.14), let $S=\left(R_{k}\right)_{M R_{k}}$. Then the following statements are equivalent:

(2.15.1) There exists an integral extension domain of $R$ which has a mcpil $n$.

(2.15.2) There exists an integral extension domain of $S$ which has a mopil $n$.

(2.15.3) There exists a minimal prime ideal $z$ in the completion of $R$ such that depth $z=n$.

(2.15.4) There exists a minimal prime ideal $w$ in the completion of $S$ such that depth $w=n$.

Proof. (2.15.1) $\Longleftrightarrow(2.15 .3)$ and (2.15.2) $\Longleftrightarrow(2.15 .4)$, by (2.3); and (2.15.3) $\Longleftrightarrow(2.15 .4)$, by (2.5). Q.E.D.

Before giving further corollaries, it should be noted that the corollaries given below are really corollaries of (2.14); that is, we do not have a built-in circle. (The proofs of the results used to prove (2.14) are not based on the corollaries.) The only place where this is not exactly true is in the reference to [6, Proposition 3.5] in the proof of (2.3). However, this reference can be replaced by [6, Lemma 2.17], and then [6, Proposition 3.5] follows from a short computation. 
(2.16) Corollary (CF. [7, Theorem 2.21(5) $\Leftrightarrow(8)]$ ). Let $(R, M), k, Q$, and $S$ be as in (2.14). Then $S$ is catenary if and only if $S$ satisfies the s.c.c.

Proof. $S$ is catenary if and only if every integral extension domain of $S$ satisfies the f.c.c. (by (2.14.4) $\Longleftrightarrow(2.14 .5)$ ) if and only if $S$ satisfies the s.c.c. Q.E.D.

(2.17) CoRollary (CF. [7, THEOREM $2.21(1) \Leftrightarrow(8)]$ ). Let $(R, M), k, Q$, and $S$ be as in (2.14). Then $R$ satisfies the s.c.c. if and only if $S$ is catenary.

Proof. $S$ is catenary if and only if every integral extension domain of $R$ satisfies the f.c.c. (by (2.14.1) $\Longleftrightarrow(2.14 .5)$ ) if and only if $R$ satisfies the s.c.c. Q.E.D.

In the following corollary we use the fact that if $p$ is a prime ideal in a local domain $R$ which satisfies the s.c.c., then $R_{p}$ satisfies the s.c.c. This is proved in (2.26) below.

(2.18) COROLlaRY (CF. [6, THEOREM 3.6]). The following statements are equivalent for a local domain $(R, M)$ :

(2.18.1) $R$ satisfies the s.c.c.

(2.18.2) $R_{k}$ is catenary, for some $k>0$.

(2.18.3) $R_{k}$ is catenary, for all $k \geqslant 0$.

PRoof. It is clear that (2.18.3) implies (2.18.2). Also, (2.18.2) implies (2.18.1), by (2.17), since if $R_{k}$ is catenary, for some $k>0$, then $S=$ $\left(R_{k}\right)_{\left(M, X_{1}, \ldots, X_{k}\right)}$ is catenary.

Finally, assume that $R$ satisfies the s.c.c. and let $k \geqslant 0$. Then to prove that $R_{k}$ is catenary, it suffices to prove that if $N$ is a maximal ideal in $R_{k}$, then $\left(R_{k}\right)_{N}$ is catenary. For this, it may clearly be assumed that $k>0$. Also, if $N \cap R=M$, then $\left(R_{k}\right)_{N}$ is catenary (by (2.17), since $N$ is maximal (hence $M R_{k} \subset N$ )), so assume that $N \cap R=P \neq M$. Then $R_{P}$ satisfies the s.c.c. and $N^{*}=$ $N R_{P}\left[X_{1}, \ldots, X_{k}\right]$ is a maximal ideal which lies over $P R_{P}$. Hence, by (2.17) applied to $R_{P},\left(R_{k}\right)_{N}=\left(\left(R_{P}\right)_{k}\right)_{N}$ is catenary. Q.E.D.

(2.19) Corollary (CF. [6, THeORem 3.1]). A local domain $R$ satisfies the s.c.c. if and only if $R$ is quasi-unmixed.

Proof. $R$ satisfies the s.c.c. if and only if, by $(2.17), R\left[X_{1}\right]_{\left(M, X_{1}\right)}$ is catenary if and only if $W\left(1,\left(M, X_{1}\right)\right)$ is a one-point set if and only if, by (2.14.2) $\Longleftrightarrow(2.14 .6)$, all minimal prime ideals in the completion of $R$ have the same depth if and only if $R$ is quasi-unmixed. Q.E.D.

In (2.26) below it will be shown that if a local domain $R$ satisfies the s.c.c., then, for each prime ideal $p$ in $R, R_{p}$ and $R / p$ satisfy the s.c.c. Using this, a 
fairly easy proof of the following known and important result can be given using (2.16) and (2.17).

(2.20) Corollary (CF. [6, CoROllaRY 3.7]). If a local domain $R$ satisfies the s.c.c., then, for each prime ideal $p$ in $R$, and for each local domain $L$ which is a locality over $R / p, L$ satisfies the s.c.c.

Proof. Assume that $R$ satisfies the s.c.c., let $p$ be a prime ideal in $R$, and let $L$ be a local domain which is a locality over $R / p$, say $L=$ $\left((R / p)\left[a_{1}, \ldots, a_{k}\right]\right)_{q}$. Then there exists a prime ideal $Q$ in $R_{k}$ such that $L$ is a homomorphic image of $\left(R_{k}\right)_{Q}$. Let $P=Q \cap R$, so $\left(R_{k}\right)_{Q}$ is a quotient ring of $A=R_{P}\left[X_{1}, \ldots, X_{k}\right]$ and $R_{P}$ satisfies the s.c.c. Therefore, if $N$ is a maximal ideal in $A$ such that $Q A \subseteq N$, then $D=A_{N}$ satisfies the s.c.c. (by (2.16) and (2.17)); hence $\left(R_{k}\right)_{Q}=D_{Q D}$ satisfies the s.c.c., and so $L$ satisfies the s.c.c. (see (2.26)). Q.E.D.

(2.9.2) says that the lengths of maximal chains of prime ideals in $\left(R_{k}\right)_{Q}$ ( $k>0$ and $M R_{k} \subset Q$ ) are known, once the lengths of maximal chains of prime ideals in $D=R\left[X_{1}\right]_{\left(M, X_{1}\right)}$ are known. It is, of course, of much interest to know how the lengths of maximal chains of prime ideals in $D$ compare to the lengths of maximal chains of prime ideals in $R$. The only case in which we know how these lengths compare is when $R$ satisfies the s.c.c.; that is, by (2.17), when $W\left(1,\left(M, X_{1}\right)\right)=$ altitude $R+1$ \}. In all other cases this is a very difficult problem, but we make the following conjecture.

(2.21) Conjecture. $\{n+1 ; n \in W(0, M)\} \subseteq W\left(1,\left(M, X_{1}\right)\right) \subseteq$ $\{n+1 ; n \in W(0, M)\} \cup\{2\}$.

The first containment is obvious.

This conjecture was called the upper conjecture in [2], and it was shown there that the depth conjecture implies the upper conjecture which, in turn, implies the catenary chain conjecture [2, Propositions 3.5 and 3.7]. (Depth conjecture: If $P$ is a prime ideal in a local domain $R$ such that height $P>1$, then there exists a prime ideal $p$ in $R$ such that $p \subset P$ and depth $p=\operatorname{depth} P+1$. Catenary chain conjecture: The integral closure of a catenary local domain satisfies the c.c.)

Nagata's examples [5, Example 2, pp. 203-205] support the conjecture. That is, in these examples, the first inclusion is an equality for $m>0$ ( $m$ as in [5]) and the second inclusion is an equality for $m=0$.

The following result shows that only the last step in a maximal chain of prime ideals keeps us from verifying the upper conjecture for a catenary local domain:

If $(R, M)$ is a catenary local domain and $(0) \subset Q_{1} \subset \cdots \subset Q_{n}$ is a maxi- 
mal chain of prime ideals in an integral extension domain of $R$, then height $Q_{i}=$ $i=$ height $Q_{i} \cap R(i<n)$. For, $R_{Q_{i} \cap R}$ satisfies the s.c.c. [8, Theorem 3.9]. (Also, height $Q_{n}=$ height $M$, if $n>1$. Now $n=1$ is possible; that is, possibly $Q_{1}=Q_{n}$ is a height one maximal ideal and altitude $R>1$, but this does not cause any real problem. The real problem lies in showing that if $n>1$, then depth $Q_{n-1}=1$.)

We close the discussion of the upper conjecture with the following result.

(2.22) COROLLARY. The following statements are equivalent:

(2.22.1) The upper conjecture holds.

(2.22.2) If $R^{\prime}$ is an integral extension domain of a local domain $R$ such that $R^{\prime}$ has a mcpil $n$, then either there exists a mcpil $n$ in $R$ or $n=1$.

(2.22.3) If there exists a depth $n$ minimal prime ideal in the completion of a local domain $R$, then there either exists a mcpil $n$ in $R$ or $n=1$.

Proof. Assume that the upper conjecture holds, let $R$ be a local do. main, and let $R^{\prime}$ be an integral extension domain of $R$. If there exists a mcpil $n$ in $R^{\prime}$, then, by (2.14.1) $\Leftrightarrow(2.14 .6), n+1 \in W\left(1,\left(M, X_{1}\right)\right) \subseteq\{i+1$; $i \in W(0, M)\} \cup\{2\}$, so (2.22.1) implies (2.22.2). Conversely, let $(R, M)$ be a local domain and let $m \in W\left(1,\left(M, X_{1}\right)\right)$. Then there exists an integral extension domain of $R$ which has a mcpil $m-1$, by $(2.14 .1) \Longleftrightarrow(2.14 .6)$. Therefore, by hypothesis, either $m-1 \in W(0, M)$ or $m-1=1$; hence (2.22.2) implies (2.22.1).

Again assume that the upper conjecture holds, let $R$ be a local domain, and let $z$ be a depth $n$ minimal prime ideal in the completion of $R$. Then, by (2.14.2) Therefore (2.22.1) implies (2.22.3). Conversely, let $(R, M)$ be a local domain and let $m \in W\left(1,\left(M, X_{1}\right)\right)$. Then there exists a depth $m-1$ minimal prime ideal in the completion of $R$, by (2.14.2) $\Longleftrightarrow(2.14 .6)$. Therefore, by hypothesis, either $m-1 \in W(0, M)$ or $m-1=1$; hence (2.22.3) implies (2.22.1). Q.E.D.

If there exists a mcpil $m$ in $D=R\left[X_{1}\right]_{\left(M, X_{1}\right)}$, then there exists a mcpil $m$ in $D$, say $(0) \subset p_{1} \subset \cdots \subset p_{m}=\left(M, X_{1}\right) D$ such that $R\left[X_{1}\right] /\left(p_{1} \cap R\left[X_{1}\right]\right)$ is integral over $R$. This follows by taking $S=D$ in the following corollary.

(2.23) Corollary. Let $(R, M), k, Q$, and $S$ be as in (2.14). Assume that there exists a mcpil $n$ in $S$. Then there exists a mcpil $m=n-(k-1)+$ depth $Q$ in $R\left[X_{1}\right]_{\left(M, X_{1}\right)}$, say $(0) \subset p_{1} \subset \cdots \subset p_{m}=\left(M, X_{1}\right)$, such that $R\left[X_{1}\right] /\left(p_{1} \cap R\left[X_{1}\right]\right)$ is integral over $R$.

Proof. By (2.14.1) $\Longleftrightarrow(2.14 .5)$, there exists an integral extension domain of $R$ which has a mcpil $m-1$. Therefore, by (2.2), there exists a principal 
integral extension domain $R[c]$ of $R$ which has a mcpil $m-1$, say (0) $\subset \cdots \subset$ $q_{m-1}=N$ such that $c \in N$. Then $N=(M, c) R[c]$, so the conclusion follows by considering the preimage of this chain in $R\left[X_{1}\right]$. Q.E.D.

(2.14) allows us to determine possible lengths of maximal chains of prime ideals in integral extension domains of $R / p$ and of $R_{p}$ (where $p$ is a prime ideal in a local domain $R$ ). This is explicitly stated in the next two remarks.

(2.24) REMARK. Let $p$ be a prime ideal in a local domain $(R, M)$, and let $D=R\left[X_{1}\right]_{\left(M, X_{1}\right)}$.

(2.24.1) There exists an integral extension domain of $R / p$ which has a mcpil $n$ if and only if in $D$, there exists a saturated chain of prime ideals of length $n+1$ with $p D$ the smallest term and $\left(M, X_{1}\right) D$ the largest term.

(2.24.2) There exists an integral extension domain of $R_{p}$ which has a mcpil $n$ if and only if in $D$ there exists a saturated chain of prime ideals of length $n+1$ with $\left(p, X_{1}\right) D$ the largest term and (0) the smallest term.

Proof. (2.24.1) There exists an integral extension domain of $R / p$ which has a mcpil $n$ if and only if in $D / p D=(R / p)\left[X_{1}\right]_{\left(M / p, X_{1}\right)}$ there exists a mcpil $n+1$, by (2.14.1) $\Longleftrightarrow(2.14 .6)$, and (2.24.1) clearly follows from this.

(2.24.2) There exists an integral extension domain of $R_{p}$ which has a mcpil $n$ if and only if in $D_{\left(p, X_{1}\right) D}=R_{p}\left[X_{1}\right]_{\left(p R_{p}, X_{1}\right)}$ there exists a mcpil $n+1$, by $(2.14 .1) \Longleftrightarrow(2.14 .6)$, and (2.24.2) clearly follows from this. Q.E.D.

(2.25) REMARK. Let $p$ be a prime ideal in a local domain $(R, M)$.

(2.25.1) If there exists an integral extension domain of $R / p$ which has a mcpil $n$, then there exists an integral extension domain of $R$ which has a mcpil $n+$ height $p$.

(2.25.2) If there exists an integral extension domain of $R_{p}$ which has a mcpil $n$, then there exists an integral extension domain of $R$ which has a mcpil $n+$ depth $p$.

ProOF. (2.25.1) If there exists an integral extension domain of $R / p$ which has a mcpil $n$, then, by (2.24.1), there exists a mcpil $n+1+$ height $p$ in $D$; hence, by $(2.14 .1) \Leftrightarrow(2.14 .6)$, there exists an integral extension domain of $R$ which has a mcpil $n+$ height $p$.

The proof of (2.25.2) is similar. Q.E.D.

(2.26) CoRollary (CF. [4, Lemma 1]). Let $p$ be a prime ideal in a local domain $R$. If $R$ satisfies the s.c.c., then $R_{p}$ and $R / p$ satisfy the s.c.c.

Proof. Assume that there exists an integral extension domain of $R / p$ which has a mcpil $n$. Then, by (2.25.1), there exists an integral extension domain of $R$ which has a mcpil $n+$ height $p$, so $n+$ height $p=$ altitude $R$, if $R$ satisfies the s.c.c., and height $p+\operatorname{depth} p=$ altitude $R$. Therefore depth $p=n$; hence $R / p$ satisfies the s.c.c. 
The proof that $R_{p}$ satisfies the s.c.c. is similar. Q.E.D.

(2.9) was stated for polynomial rings over a local domain. However, it is straightforward to see that it holds for polynomial rings over a Noetherian domain. Specifically, we close this paper with the following remark.

(2.27) REMARK. Let $P$ be a prime ideal in a Noetherian domain $A$, and let $Q$ be a prime ideal in $A_{k}=A\left[X_{1}, \ldots, X_{k}\right]$ such that $Q \cap A=P$. Let $B=$ $\left(A_{k}\right)_{(A-P)}$, and let $d=\operatorname{depth} Q B$, so $\operatorname{trd}(B / Q B) /\left(A_{P} / P A_{P}\right)=d$. Renumber the $X_{i}$ such that $X_{1}, \ldots, X_{d}$ modulo $Q B$ are a transcendence basis for $B / Q B$ over $A_{P} / P A_{P}$, and let $W_{i}=\left\{n_{i}\right.$; there exists an integral extension domain of $\left(A_{i}\right)_{Q \cap A_{i}}$ which has a mcpil $\left.n_{i}\right\}\left(i=0,1, \ldots, d\right.$ with $\left.A_{0}=A\right)$. Then $W_{0}=\cdots=w_{d}$ and $n \in W_{d}=W_{0}$ if and only if $n+j \in W\left(d+j, Q \cap A_{d+j}\right)(j=1, \ldots$, $k-d)$.

Proof. By [7, Lemma 4.2], $Q \cap A_{i}=P A_{i}(i=0,1, \ldots, d)$; hence the $W_{i}$ are equal (2.9.1). Let $C=\left(A_{d}\right)_{P A}$, and let $C_{j}=C\left[X_{d+1}, \ldots, X_{d+j}\right]$ $\left(j=0,1, \ldots, k-d\right.$ and $\left.C_{0}=C\right)$. Then $\left(A_{k}\right)_{Q}=\left(C_{k-d}\right)_{Q^{\prime}}$, where $Q^{\prime}=$ $Q\left(A_{k}\right)_{Q} \cap C_{k-d}$, so, since $\left(Q \cap A_{d+j}\right) A_{d+j+1} \subset Q \cap A_{d+j+1} \quad(j=0,1, \ldots$, $k-d-1)$, the ideals $Q^{\prime} \cap C_{j}$ are maximal $(j=0,1, \ldots, k-d)$. Therefore $n \in W_{d}$ if and only if $n+j \in W\left(d+j, Q \cap A_{d+j}\right)(j=1, \ldots, k-d)$, by (2.14.1) $\Longleftrightarrow(2.14 .5)$ applied to $C\left(\left(A_{d+j}\right)_{Q \cap A_{d+j}}=\left(C_{j}\right)_{Q^{\prime} \cap C_{j}}\right)$. Q.E.D.

\title{
BIBLIOGRAPHY
}

1. S. McAdam, Saturated chains in Noetherian rings, Indiana Univ. Math. J. 23 (1973/74), 719-728. MR 48 \#11094.

2. S. McAdam and E. G. Houston, Chains of primes in Noetherian rings, Indiana Univ. Math. J. 24 (1975), 741-753.

3. M. Nagata, On the chain problem of prime ideals, Nagoya Math. J. 10 (1956), 51-64. MR 18, 8 .

4. - Note on a chain condition for prime ideals, Mem. Coll. Sci. Univ. Kyoto Ser. A. Math.32 (1959), 85-90. MR 21 \#7227.

5. - Local rings, Interscience Tracts in Pure and Appl. Math., no. 13, Interscience, New York, 1962. MR 27 \#5790.

6. L. J. Ratliff, Jr., On quasi-unmixed local domains, the altitude formula and the chain condition for prime ideals. I, Amer. J. Math. 91 (1969), 508-528. MR 40 \#136.

7. On quasi-unmixed local domains, the altitude formula, and the chain condition for prime ideals. II, Amer. J. Math. 92 (1970), 99-144. MR 42 \#249.

1108. MR 45 \#6804.

8. - Characterizations of catenary rings, Amer. J. Math. 93 (1971), 1070-

9. - Chain conjectures and H-domains, Conf. on Commutative Algebra (Univ. Kansas, Lawrence, Kan., 1972), Lecture Notes in Math., vol. 311, Springer-Verlag, Berlin, 1973, pp. 222-238. MR 49 \#2714. 75-93.

10. - Four notes on saturated chains of prime ideals, J. Algebra 39 (1976),

11. O. Zariski and P. Samuel, Commutative algebra, Vol. II, University Ser. in Higher Math., Van Nostrand, Princeton, N. J., 1960. MR 22 \#11006.

\author{
DEPARTMENT OF MATHEMATICS, UNIVERSITY OF CALIFORNIA, RIVER- \\ SIDE, CALIFORNIA 92502
}

DEPARTMENT OF MATHEMATICS, UNIVERSITY OF TEXAS, AUSTIN, TEXAS 